\title{
Wie verbindlich ist das Wissen vom Gene Editing? Ethische Reflexionen zu einem aktuellen Normierungsprozess
}

\author{
Stefanie Kiewitt-Straßburg
}

Online publiziert: 8. Oktober 2019

(C) Der/die Autor(en) 2019, korrigierte Publikation 2019

Zusammenfassung Aktuelle Untersuchungen zum Gene Editing an menschlichen Embryonen zeigen große Erfolge bei der Korrektur vererbbarer Krankheiten und treiben die Grundlagenforschung sowie die Präimplantations-Gendiagnostik enorm voran. Was von Forschungen dieser Art aus ethischer Sicht zu halten ist, ist dagegen weniger eindeutig. Ein Blick auf den aktuellen Diskurs zeigt, dass diese Entwicklungen sehr kontrovers diskutiert werden und eine Einigung darüber, ob sie vorangebracht werden sollen oder nicht, keineswegs in Sicht ist. Zum Zweck der Orientierung und auch besseren Positionierung in der aktuellen Debatte befasst sich die vorliegende Untersuchung zum einen mit der Art des Wissens vom Gene Editing sowie dessen epistemischen Grundlage und zum anderen mit den ethisch-normativen Überzeugungen, an die die Befürworter und Gegner in der ethischen Bewertung des Gene Editing jeweils appellieren. Dabei zeigt sich, dass die Überzeugungen, auf die sich die Befürworter stützen, der speziellen Art dieses Wissens und seiner epistemischen Grundlage stärker Rechnung tragen, während das von den Überzeugungen, auf die sich die Gegner berufen, gerade nicht zu erwarten ist. Diese Einsicht in die Struktur der Debatte erlaubt es, die verschiedenen Argumentationsweisen in Beziehung zu setzen, ihre jeweiligen Perspektiven einzunehmen und auch ihre blinden Flecken aufzudecken.

Schlüsselwörter Gene Editing · CRISPR-Cas9 · Wissen · Verbindlichkeit · Bioethik · Epistemologie 
Abstract Recent studies of embryonic gene editing show great successes in the correction of germline mutations and make tremendous progress in basic research and pre-implantation genetic diagnosis. How this research is ethically assessed, however, is less clear. A look at the current discourse shows that these developments are discussed controversially. An agreement on whether or not such researches should be continued is by no means in sight. For the purpose of orientation, the present study deals with the nature of the knowledge of gene editing and its epistemic basis. Furthermore, it examines the ethical-normative convictions to which the proponents and opponents refer in the ethical evaluation of gene editing. It shows that the beliefs on which the advocates are based take greater account of the specific nature of this knowledge and its epistemic basis. In contrast, this is not to be expected from the beliefs invoked by the adversaries. This insight into the structure of the controversy allows to contrast the different ways of argumentation, to take their respective perspectives into account and to reveal their blind spots.

\section{Inhaltsverzeichnis}

1 Gene Editing der menschlichen Keimbahn

2 Wissen vom Gene Editing

2.1 Wissen und Verbindlichkeit

2.2 Das verbindliche Fundament des Wissens vom Gene Editing

2.3 Ethische Einwände gegen das Gene Editing und das Paradox einer wertfreien Verbindlichkeit

3 Das Wissen vom Gene Editing als prekäres Wissen

\section{Gene Editing der menschlichen Keimbahn}

Das Fachmagazin Nature veröffentlichte im August 2017 einen Artikel zum Gene Editing an menschlichen Embryonen, ein Eingriff in die menschliche Keimbahn in vitro. Der Artikel beschreibt die Korrektur der heterozygoten MYBPC3-Mutation zum Zeitpunkt der Befruchtung durch die gezielte, CRISPR-Cas9 basierte Genmodifikation. Auswirkung dieser Mutation ist die ,familiäre hypertrophe Kardiomyopathie“, eine dominante Erbkrankheit der Herzmuskelschwäche. Das Bemerkenswerte an dem Verfahren ist, dass die Reparatur mit einer umso höheren Wirksamkeit auftrat, desto früher der jeweilige Schnitt des Erbgutstrangs mithilfe des CRISPRCas9-Werkzeugs veranlasst wurde, nämlich in der Metaphase II, in der das Ei den Eierstock verlässt und gewöhnlich noch nicht befruchtet, aber reif zur Befruchtung ist: Durch die Co-Injektion des Spermiums und der CRISPR-Cas9-Komponenten in die Eizelle während der Metaphase II ließ sich die oft auftretende Mosaikbildung bei der nun stattfindenden Zellteilung zu einem großen Teil vermeiden, in welcher der Embryo eine Variation von mutierten und nicht mutierten Zellen aufweist. Auf diese Weise konnte auch ein hoher Ertrag homozygoter Embryonen ohne Anzeichen auf Off-Target-Mutationen erreicht werden, welche das biologische Risiko von CRISPR-Cas9 basierten Korrekturverfahren darstellen. Sie treten auf, wenn durch das CRISPR-Cas9-Werkzeug an Stellen im Genom geschnitten wird, die eigentlich 
nicht vorgesehen waren. Der Eingriff in das menschliche Erbgut, so das Fazit der Studie, wird damit umso sicherer, je früher er geschieht, hier zum Zeitpunkt der Befruchtung während der Metaphase II (Ma 2017).

Mithilfe der CRISPR-Cas9-Technologie ist es möglich, die menschliche Erbinformation präzise, schnell und preiswert zu bearbeiten. Was aber ist von Forschungen dieser Art aus ethischer Sicht zu halten? Die Grundlagenforschung verspricht ein Verfahren, das durch seine Effizienz, Präzision und Sicherheit ein hohes Potential zur Nutzung der Technologie für die Korrektur vererbbarer Mutationen in menschlichen Embryonen aufweist. Natürlich bleibt noch vieles vor der klinischen Anwendung zu überlegen, z. B. zur Anwendbarkeit der Technik in Hinblick auf die Korrektur auch anderer vererbbarer Mutationen, so das Forscherteam um Shoukrat Mitalipov und Hong Ma, den Leitern der oben angeführten Untersuchung (ebd., S. 1). Dennoch sind die Grundlagenforschung zur Vererbbarkeit schwerer Krankheiten sowie die Präimplantations-Gendiagnostik mit diesen Forschungen ein gutes Stück vorangebracht und das obwohl in der Frage, ob wir die menschliche Keimbahn überhaupt genetisch verändern wollen, dürfen oder sollen, alles andere als Einigkeit herrscht. Der Blick auf die aktuelle bioethische Debatte um die ethische Bewertung der CRISPRCas9-Technologie und des Gene Editing insgesamt zeigt, dass diese Entwicklungen zum Teil auf das Schärfste verurteilt werden, zum Teil aber auch offen begrüßt oder zumindest akzeptiert und gegen kritische Stimmen verteidigt werden. Die verschiedenen Positionen lassen sich danach differenzieren, ob sie außer der Forschung am menschlichen Genom auch der Anwendung dieser Technologien zustimmen und ob diese Anwendung auf medizinisch-therapeutische Zwecke zu beschränken ist oder auch einen prospektiven, gestalterischen Gebrauch zulässt, durch den sich das Aussehen oder die kognitiven Fähigkeiten eines Embryos optimieren lassen. Wiederum umstritten ist, ob sich diese Kriterien in der Praxis bewähren könnten (slipperyslope-Argumente; s. u.).

Ob Befürwortung oder Ablehnung, eines scheint gewiss: Die Debatte um das Gene Editing drängt zur Beteiligung, da das rasante Voranschreiten der Entwicklungen sowie der Einfluss, den diese neue Technologie auf unser gemeinsames Leben, unser Selbstverständnis als Menschen nehmen kann und wird, nicht zu unterschätzen sind. Für eine solche Beteiligung an der Debatte ist es wichtig, sich im bestehenden Diskurs zum Gene Editing auszukennen. Um eine solche Orientierung zu gewinnen, kann es hilfreich sein, sich zum einen die Art des Wissens vom Gene Editing sowie dessen epistemische Grundlage zu vergegenwärtigen und zum anderen die ethischnormativen Überzeugungen in den Blick zu nehmen, an die die Befürworter und Gegner in der ethischen Bewertung des Gene Editing jeweils appellieren. Denn die Vermutung liegt nahe, dass die Überzeugungen, auf die sich die Befürworter stützen, der speziellen Art dieses Wissens und seiner epistemischen Grundlage stärker Rechnung tragen, während das von den Überzeugungen, auf die sich die Gegner berufen, gerade nicht zu erwarten ist. Dieses Verhältnis zu sehen zwischen der epistemischen Grundlage, auf der das Wissen vom Gene Editing beruht, und den jeweiligen für verbindlich erachteten ethischen Überzeugungen, die für dessen Befürwortung oder Ablehnung angeführt werden, kann für ein Verständnis der Debatte hilfreich und für die kritische Bildung eines eigenen moralischen Urteils zum Gene Editing entschei- 
dend sein. Die Absicht des vorliegenden Aufsatzes ist es, in dieser Hinsicht Klarheit zu schaffen.

Der Aufsatz ist wie folgt aufgebaut: Um den Wissensbegriff zu klären, wird in einem ersten Schritt Wittgensteins Konzeption von Wissen und Gewissheit erläutert. Sie bietet sich an, weil darin nach den Voraussetzungen von Wissen gefragt wird und die Begriffe „Wissen“ und „Verbindlichkeit“ im Zusammenhang thematisiert werden (Abschn. 2.1). Um sowohl das Wissen vom Gene Editing als auch dessen Voraussetzungen anhand dieses epistemologischen Grundmodells bestimmen zu können, ist es erforderlich, sich über deren kulturhistorischen Kontext Aufschluss zu geben, wofür Husserls Krisis-Schrift herangezogen wird. Der Rekurs auf die beiden Autoren geschieht ausschließlich in heuristischer, problemerschließender Absicht und hat keinerlei werkexegetischen oder -komparativen Anspruch. ${ }^{1}$ Mit Husserl soll vor allem gezeigt werden, dass das positive Wissen vom Gene Editing einen verbindlichen Kern aufweist, insofern es sich in das positivistische und technisch-rationalistische Weltbild einfügt, und dass dieses Weltbild auch von bestimmten normativ-ethischen Bewertungen des Gene Editing in der aktuellen bioethischen Debatte geteilt wird. Das betrifft vor allem einzelne Argumente, die zur Befürwortung des Gene Editing herangezogen werden (Abschn. 2.2). Auf der anderen Seite wird dieses Wissen vom Gene Editing als normabweichend bzw. normüberschreitend gekennzeichnet, insofern es im gegenwärtigen bioethischen Diskurs, und zwar vor allem von den Gegnern des Gene Editing, mit bestimmten ethischen Überzeugungen kontrastiert wird, die unser Leben unabhängig von den verbindlichen Ansprüchen dieses positivistischen Weltbilds prägen (Abschn. 2.3).

Aufgrund dieser Ambivalenz, sich bruchlos in ein fest etabliertes Weltbild zu fügen und doch mit bestimmten, davon unabhängigen, aber nicht weniger anerkannten Normen in Konflikt zu geraten, kann das Wissen vom Gene Editing abschließend

\footnotetext{
${ }^{1}$ Es leuchtet nicht unmittelbar ein, zwischen Husserl und Wittgenstein eine derartige Verbindung herzustellen. Während Husserl als Vertreter der traditionellen Phänomenologie darum bemüht ist, in der logischen Reflexion endgültige Regeln oder Wahrheiten aufzudecken, geht es Wittgenstein als Vertreter der analytischen Philosophie lediglich um die Beschreibung und Feststellung logischer Sachverhalte. Trotz aller Unterschiede besteht die Gemeinsamkeit zwischen den beiden Denkern aber vor allem darin, ,daß beide eine logische Analyse geben, welche nicht auf faktisch Gegebenem beruht. Die Weltstruktur ist bei beiden eine logische, eine Struktur aller möglichen Welten. [...] Die Entwicklung Husserls könnte man dadurch kennzeichnen, daß er seine phänomenologischen Analysen innerhalb des Horizonts der sogenannten Lebenswelt situiert. [...] [Bei] Wittgenstein [werden] die Sprache mit einem Spiel und seinen Spielregeln, wie z. B. dem Schachspiel, verglichen [...] und die logischen Regeln also der Reibung des konkreten Lebens angepaßt [...].“(Peursen 1965, S. 127 ff.; vgl. auch Bermes, 2017, S. 103) - Sabine Knabenschuh hebt treffend hervor, dass Phänomenologie bei Wittgenstein wesentlich als Grammatik auftritt: „Eine Grammatik, die zum Erfahrungsprinzip wird, insofern sie eine Übersichtlichkeit unserer epistemisch-grammatikalischen Mechanismen ermöglicht, da sich diese im Artikulieren der unmittelbaren Erfahrung auf die internen Relationen beschränken, also auf unsere wesentlich lebenswichtigen Wechselbeziehungen mit der Welt. Eine Grammatik, die holistisch ist, da jede von internen Relationen erstellte ,grammatische Umgebung' einen elementaren Kontext bildet, dessen Dynamik alle kombinatorische, situative oder historische Kontextualität trägt. Eine Grammatik schließlich, deren Schwerpunkt darin liegt, daß sich die Standortbestimmungen bei unseren Annäherungen an die Lebenswelt aus stets verschiedenen Positionen, Winkeln oder Perspektiven vollziehen, kurz, auf parallaktische Art vonstatten gehen. [...] Von dieser Eigenheit abgesehen, besteht der wesentliche Unterschied zwischen traditioneller Phänomenologie und der wittgensteinschen darin, daß letztere nicht als Programm zu verstehen ist, sondern als eine Einstellung unseren lebensweltlichen Beziehungen gegenüber.“ (Knabenschuh 2013, S. 175 ff.).
} 
als prekäres Wissen bezeichnet werden. Als solches ist es Teil eines Normalisierungs- und Normierungsprozesses, der durch die wissenschaftliche Forschung, die praktische Anwendung und die ethische Bewertung vorangetrieben wird und dessen Ausmaß und konkrete Gestalt noch vollkommen offen sind (Abschn. 3).

\section{Wissen vom Gene Editing}

\subsection{Wissen und Verbindlichkeit}

Um dem Zusammenhang von „Wissen“ und „Verbindlichkeit“ philosophisch auf die Spur zu kommen, bietet es sich an, auf Wittgensteins Konzeption von „Gewissheit“ zurückzugreifen (Wittgenstein 1984 [1970]). Denn jedes positive Wissen basiert auf Gewissheiten, die sich ihrerseits durch eine bestimmte Verbindlichkeit auszeichnen.

Wittgensteins Überlegungen setzen beim Sprechen als einer regelgeleiteten Praxis des Menschen an, durch die die menschliche Welt mitsamt ihren Gegenständen und Sachverhalten konstituiert wird. Im Sprechen spielen wir sogenannte Sprachspiele, deren inhärente Regeln wir teilen, sofern wir zu der Sprachgemeinschaft gehören, die diese Sprachspiele spielt. Sprachspiele sind mit unserem gewöhnlichen Tun, unserem alltäglichen Verhalten sowie mit unseren Überzeugungen und Wertvorstellungen verknüpft. Im Rahmen einer geteilten Sprachpraxis „bewährt sich“ (ebd., § 474, S. 215) ein Sprachspiel, darüber hinaus lässt es sich nicht rechtfertigen: „Ich meine: Es ist nicht begründet. Nicht vernünftig (oder unvernünftig). Es steht da - wie unser Leben.“ (ebd., § 559, S. 232)

Einige dieser Sprachspiele lassen sich als Sprachspiele des Wissens bezeichnen, wenn darin eine Behauptung über die Welt gemacht wird, mit der der Anspruch einhergeht, wahr zu sein. Wittgenstein geht es nun darum, dieses positive „Wissen“ von seinen zugrunde liegenden Überzeugungen, den „Gewissheiten“, zu unterscheiden und beide in ihrer Eigenart sowie in ihrer Beziehung zueinander aufzuklären: "Wissen“ ist in propositionaler Form verfasst und muss daher artikuliert werden. Es ist irrtumsanfällig und kann dementsprechend auch von anderen angezweifelt werden, weshalb derjenige, der einen Wissensanspruch erhebt, in der Lage sein muss, einen Weg anzugeben, der eine Überprüfung von dessen Richtigkeit erlaubt; "Wissen“ ist also potentiell begründungsbedürftig und zugleich ist es auch überhaupt begründungsfähig. All diese Kriterien treffen auf „Gewissheiten“ nicht oder zumindest nicht ohne Weiteres zu. Gewissheiten gelten auch unausgesprochen und werden überhaupt nur bei besonderen Gelegenheiten (sozusagen im Zweifelsfall) thematisch und artikuliert. Man kann sich nicht von ihrer Richtigkeit überzeugen, sie nicht begründen, nicht bezweifeln und sich auch nicht darin irren (Krebs 2007, S. 30-37). Gewissheiten bilden die unausgesprochene epistemische Basis unseres Handelns, Sprechens und eben auch Wissens; man könnte ebenso sagen: unseres Lebens.

Damit ist die Beziehung zwischen Wissen und Gewissheiten angesprochen, die als Fundierungsverhältnis verständlich wird. Erweist sich ein Wissensanspruch als problematisch, wird zu seiner Begründung in der Regel der epistemische Zusammenhang expliziert, in dem dieses Wissen seinen Platz hat; man vergewissert sich 
folglich bei dem, was unstrittig ist. Wissen ist somit rückgebunden in einen diffusen Zusammenhang von Selbstverständlichkeiten, der nicht im Ganzen, sondern immer nur in Aspekten und entsprechend der situativen Anforderungen artikuliert werden kann. Wittgenstein spricht auch von einem „Nest von Sätzen“ (Wittgenstein 1984 [1970], § 225, S. 164), die sich gegenseitig stützen und zusammen ein ,System unsrer Verifikation“ (ebd., § 279, S. 174) ausmachen. In diesem Sinne bilden Gewissheiten das Bezugssystem des Wissens (ebd., § 83, S. 136), sie ,konzeptualisieren die Welt“ (Kober 1993, S. 208) und gehören zu den „normativen Fundamenten“ (ebd., S. 200f.) der entsprechenden Sprachspiele. Sie sind selbst weder wahr noch falsch, sondern stellen vielmehr die Wahrheitsbedingungen jener Sprachspiele dar.

Es zeigt sich nun auch, inwieweit Gewissheiten ,verbindlich“ sind, nämlich insofern sie den gemeinsamen Hintergrund abgeben, vor dem die Differenzen in den Urteilen überhaupt erst sichtbar und dann auch ausdiskutiert werden können. Wird dieser Hintergrund von den Diskussionspartnern nicht anerkannt oder geteilt, kann eine Auseinandersetzung gar nicht in Gang kommen, weil es weder eine gemeinsame Konzeption von Welt, ihren Gegenständen und Sachverhalten gibt noch ein Einverständnis darüber, nach welchen Wahrheitskriterien sich Behauptungen über diese aufstellen und begründen lassen.

Es wurde gesagt, Gewissheiten bilden das epistemische Fundament unseres Lebens. Wittgenstein ist sich durchaus im Klaren darüber, dass sich dieses Leben mitsamt den zugehörigen Handlungsweisen und Sprachspielen ändern kann (Wittgenstein 1984 [1970], § 256, S. 170) und dass auch Gewissheiten von diesem Wandel nicht ausgenommen sind: „Aber was Menschen vernünftig oder unvernünftig erscheint, ändert sich.“ (ebd., § 336, S. 185) Zwar scheint er mit einem Kern relativ robuster Gewissheiten zu rechnen, die einen quasi anthropologischen Status haben und mit unserer menschlichen Lebensform (ebd., § 358, S. 191) einhergehen, wie „Das ist eine Hand.“ oder niemand ist „ohne Eltern auf die Welt gekommen“ (ebd., $\S 335$, S. 185), aber selbst diese können ins Wanken geraten. ${ }^{2}$ Das gilt erst recht für bestimmte sozio-kulturelle Überzeugungen, die ein bestimmtes Weltbild konstituieren, wie es auch den modernen Wissenschaften zugrunde liegt. Das epistemische Fundierungsverhältnis gilt auch hier: „Aber mein Weltbild habe ich nicht, weil ich mich von seiner Richtigkeit überzeugt habe; auch nicht, weil ich von seiner Richtigkeit überzeugt bin. Sondern es ist der überkommene Hintergrund, auf welchem ich zwischen wahr und falsch unterscheide." (ebd., § 94, S. 139) Jedoch ist die historische Persistenz derartiger Gewissheiten mitunter sehr viel eingeschränkter, wie in Wittgensteins berühmter Flussbettmetapher zum Ausdruck kommt: „Man könnte

\footnotetext{
2 Die Gewissheit, niemand kommt ohne Eltern auf die Welt, wird brüchig, wie das Beispiel eines im Frühjahr 2018 geborenen Babys demonstriert, das vier Jahre nach dem tödlichen Autounfall seiner chinesischen Eltern geboren wird. Kurz vor dem Unfall haben sich die Eltern einer Kinderwunschbehandlung unterzogen. Die durch das Verfahren der künstlichen Befruchtung erzeugten Embryonen blieben nach dem Unfall zurück. Durch eine Leihmutter aus Laos wurde das Baby vier Jahre später ausgetragen und wird nun von seinen Großeltern groß gezogen (Spiegel Online 2018). - „Mittels Eizell-, Samen- und Embryospende sowie Ersatz- und Leihmutterschaft kann ein Kind von verschiedenen Personen genetisch gezeugt, ausgetragen und aufgezogen werden. Da diese Verfahren im Einzelfall bis zu fünf Personen Elternstatus verschaffen (genetischer Vater/genetische Mutter/austragende Mutter/aufziehender Vater/aufziehende Mutter) und entsprechend um Elternschaftsrechte schon juristisch gestritten wurde, stellt sich die Frage, ob sich ein einfaches moralisches Kriterium für Elternschaft angeben lässt.“ (Wiesemann 2011, S. 246).
} 
sich vorstellen, daß gewisse Sätze von der Form der Erfahrungssätze erstarrt wären und als Leitung für die nicht erstarrten, flüssigen Erfahrungssätze funktionierten; und daß sich dies Verhältnis mit der Zeit änderte, indem flüssige Sätze erstarrten und feste flüssig würden.“ (ebd., § 96, S. 140), ,[I]ch unterscheide zwischen der Bewegung des Wassers im Flußbett und der Verschiebung dieses; obwohl es eine scharfe Trennung der beiden nicht gibt.“ (ebd., § 97, S. 140)

Welche Einsichten lassen sich daraus nun für das Thema Gene Editing gewinnen? Hält man sich die oben erwähnte Studie zur Korrektur jener vererbbaren Herzmuskelschwäche vor Augen, handelt es sich dabei im Sinne Wittgensteins offensichtlich um ein spezifisches medizinisch-biologisches „Wissen“ über den zellulären Aufbau von Ei- und Spermienzellen, den Vorgang der Befruchtung, die Funktionsweisen zelleigener Reparaturmechanismen im Falle von viralen Angriffen und die technischen Möglichkeiten einer CRISPR-Cas9 basierten Genmodifikation. Dieses positive Wissen wird tagtäglich und weltweit in entsprechenden Laboren angewandt, an Universitäten gelehrt sowie in Experimenten überprüft und weiterentwickelt. Weniger offensichtlich sind die dazugehörigen selbstverständlichen Überzeugungen, die den wissenschaftsinternen Diskussionen und Forschungen als verbindlicher Hintergrund zugrunde liegen. Klar ist, dass diese nicht selbst Gegenstand dieser medizinischbiologischen Wissenschaften sind und somit auch nicht durch Erfahrung, z. B. experimentell bestätigt oder widerlegt werden können. Um das verbindliche Fundament freizulegen, ist daher die Regel gesucht, nach der das wissenschaftliche Wissen geprüft und beurteilt werden kann (ebd., § 98, S. 140). Denn, so fragt auch Wittgenstein: „Kann ein Behauptungssatz, der als Hypothese funktionieren könnte, nicht auch als ein Grundsatz des Forschens und Handelns gebraucht werden? D.h. kann er nicht einfach dem Zweifel entzogen sein [...]? Er wird einfach als eine Selbstverständlichkeit hingenommen, nie in Frage gezogen, ja vielleicht nie ausgesprochen.“ (ebd., § 87, S. 137) Um hier weitere Klarheit zu erlangen, gilt es, sich im Folgenden Husserls kulturphilosophischen Überlegungen zu einer Krisis der europäischen Wissenschaften zuzuwenden.

\subsection{Das verbindliche Fundament des Wissens vom Gene Editing}

Nach Husserl hat die Idee der europäischen Wissenschaft seit Beginn der Neuzeit eine positivistische Reduktion ,auf bloße Tatsachenwissenschaft“ erfahren (Husserl 1962 [1954], § 2, S. 3). Das Erkenntnisideal besteht hierbei in der experimentellen Überprüfung von Gesetzmäßigkeiten, welche bereits vor dem Experiment definiert sind und im Experiment von der Erfahrung bestätigt werden (ebd., § 9 c, S. 32 f.; $\S$ 9f, S. 43). Der Inbegriff dieser modernen Kunstfertigkeit, so Husserl, ist die exakte Naturwissenschaft, sofern sich das wissenschaftliche Handeln an „Limesgestalten“ der „Objektivität“ oder „Exaktheit“ orientiert, welche uns zur Selbstverständlichkeit, zum Kulturobjekt ähnlich der „Zange“ geworden sind (ebd., § 9 a, S. 23 ff.). Was jenes wissenschaftliche Tun prinzipiell ausschließt, sind „Fragen nach Sinn oder Sinnlosigkeit dieses ganzen menschlichen Daseins“. (ebd., § 2, S. 4) Es wird daher bewusst das Modell von Versuch und Irrtum zugrunde gelegt, ohne das Ziel oder den Zweck dieser Tätigkeit selbst noch einmal zu reflektieren. Erkenntnis wird schlicht auf die Auslegung von positiven Befunden begrenzt, die uns als bloße Tatsa- 
chen vollkommen unabhängig von ethischen Fragen, metaphysischen oder religiösen Prämissen gegeben sind (ebd., § 3, S. 6). Die damit einhergehende Art von technischer Rationalität hat einen Zwangscharakter angenommen und der Mensch, so Husserl, ist zum „Geometer“, zum Weltvermesser, geworden (ebd., § 9 a, S. 23). Diese Diagnose führt Husserl zu dem kulturkritischen Befund, dass sich die Wissenschaften mit jener positivistischen Einschränkung der Wissenschaftsidee von der Lebenswelt - „die einzig wirkliche, die wirklich wahrnehmungsmäßig gegebene, die je erfahrene und erfahrbare Welt“ (ebd., § 9h, S. 49) - abgekoppelt haben. Anstelle der realen Praxis, in der die Dinge ,im Schwanken des bloß Typischen“ (ebd., § 9 a, S. 22) stehen, ist eine ,ideale Praxis eines ,reinen Denkens““ (ebd., § 9 a, S. 23) getreten, das sich im Reich jener reinen, allerdings nie zu erreichenden Limes-Gestalten bewegt. Darum kann diese positivistische Einschränkung der Wissenschaftsidee nur Hypothese sein und muss Hypothese bleiben, wenngleich wir sie als selbstverständlich setzen, denn sie hat sich, so Husserl, ,,bewährt“ und ist somit trotz aller Distanz zugleich Teil unserer Lebenswelt (ebd., § 9d, S. 38; § 9 e, S. 41; $\S 9$ f, S. 36-44).

Wollen wir nun die Regel verständlich machen, nach der unser Wissen vom Gene Editing geprüft und beurteilt wird, dann ist es diese auf den Positivismus reduzierte Wissenschaftsidee, die als das verbindliche Fundament dieses Wissens deutlich wird. So ist das wissenschaftliche Handeln auf dem Forschungsfeld des Gene Editing häufig darum bemüht, aufgrund des gängigen Vorwurfs der Fehleranfälligkeit der CRISPR-Cas9-Technologie eine hundertprozentige Sicherheit der Technik zu erreichen, etwa in oben genannter Studie über die Möglichkeit einer Korrektur jener Herzmuskelschwäche bei Embryonen. Fraglich bleibt allerdings, ob eine solche Sicherheit je zu erreichen ist, haben wir es doch mit dem technischen Eingriff in sich selbst organisierende Systeme zu tun. Auf dem Fundament der für selbstverständlich gehaltenen positivistischen Wissenschaftsidee wird jedoch von einer im Experiment demonstrierbaren ,Sicherheit“ ausgegangen, sobald sich das Ereignis bzw. der jeweilige, sich im Experiment zeigende Befund unter den gleichen Bedingungen wiederholt. Diese Vorstellung von der objektiven Aussagekraft eines Experiments verschafft dem wissenschaftlichen Vorgehen eine induktive Voraussicht, sodass vermeintlich unmessbare und unbekannte Gestaltvorkommnisse plötzlich doch berechenbar werden (ebd., § 9 b, S. 31).

Husserl hebt hervor, dass jene Selbstverständlichkeit des positivistisch reduzierten Wissenschaftsideals nicht nur zur Abkopplung der Wissenschaften von der Lebenswelt geführt, sondern sie sich zugleich wie ein Ideenkleid über die gesamte Lebenswelt gelegt hat (ebd., §9h, S. 52). Unser alltägliches Denken und Urteilen ist darum häufig von dieser impliziten Grundüberzeugung gefärbt, ohne dass wir uns explizit auf sie beziehen. Dieses Denken muss darum nicht selbst schon positivistisches Denken sein. Auch die auf Sinn und Zweck ausgerichtete Bildung eines moralischen Urteils kann mit der fraglosen Anerkennung jener Limesgestalten von Objektivität und Exaktheit einhergehen und ebenso kann eine ethisch-normative Position zum Gene Editing von der Selbstverständlichkeit getragen sein, es mit

\footnotetext{
${ }^{3}$ Husserls „Lebenswelt“-Begriff wird kontrovers diskutiert und ist schon bei Husserl selbst alles andere als eindeutig (Orth 2000).
} 
einem objektiven und neutralen Wissen zu tun zu haben, selbst wenn sie sich ihrem Selbstverständnis nach auf ganz andere Grundüberzeugungen stützt. Symptomatisch dafür ist etwa das Plädoyer für „Forschungsfreiheit“ in Hinblick auf das Gene Editing, wonach die Forschungen zum Gene Editing ganz unabhängig von ihren therapeutischen Anwendungen zu wegweisenden, für die Gesellschaft extrem wichtigen Erkenntnissen führen sollen und daher zu befürworten sind. Dieses Konzept verträgt sich weitgehend mit der Gewissheit, dass diese Forschungen ein objektives und exaktes Wissen über bestimmte biologische Zusammenhänge erzeugen und auch nur in dieser Hinsicht eine bindende Verpflichtung aufweisen. Dieses Wissen hat schließlich enormes Potential für das weitere Erforschen von Therapieansätzen, etwa zur Behandlung von Krankheiten. Gegenüber seiner tatsächlichen Anwendung zur Therapie werden die zugrunde liegenden Forschungen aber als unabhängig, unverbindlich bzw. als wertneutral angesehen. ${ }^{4}$ Zur Logik dieser Forderung für die Forschungsfreiheit gehört daher eine bestimmte, eben positivistisch-reduzierte Auffassung des gewonnenen Wissens, das von sich aus nicht schon zu seiner Anwendung drängt, sondern lediglich zur freien Verfügung steht, um für äußere Zwecke eingespannt zu werden. Eine solche Position für die Forschungsfreiheit verteidigten etwa die Interdisziplinäre Arbeitsgruppe Gentechnologiebericht der Berlin-Brandenburgischen Akademie der Wissenschaften im Sommer 2015 (Berlin-Brandenburgische Akademie der Wissenschaften, 2017 [2015]) und die Nationale Akademie der Wissenschaften Leopoldina 2017 (Nationale Akademie der Wissenschaften Leopoldina 2017). Die Akademie-Mitglieder stammen sowohl aus den Natur- wie aus den Geisteswissenschaften. In ihrem jeweiligen Papier betonen sie die Notwendigkeit der Forschung an der menschlichen Keimbahn, freilich unter Beachtung geltender rechtlicher und ethischer Regeln. Was die rechtliche Lage betrifft, so sollen überschüssige Embryonen künftig in Deutschland für hochrangige Forschungszwecke verwendet werden dürfen, ${ }^{5}$ insbesondere zur Entwicklung neuer Therapieansätze für genetische Erkrankungen (Nationale Akademie der Wissenschaften Leopoldina 2017, S. 8 f.). In Hinblick auf konkrete Keimbahninterventionen fordern sie dagegen ein internationales Moratorium, um weitere Forschungen voranzubringen, durch die noch vorhandene Unwägbarkeiten und Risiken minimiert werden sollen. ${ }^{6}$

Der hier getroffenen politischen Entscheidung, dem ,Ja“ zur Forschung und dem „Nein“ zur Anwendung, geht eine utilitaristische Risiko-Nutzen-Abwägung voraus: „Jede gezielte Keimbahnveränderung mit Auswirkungen auf einen später geborenen Menschen sollte beim derzeitigen Stand der Forschung unterbleiben. Bevor ein Ein-

\footnotetext{
${ }^{4}$ Vgl. die Stellungnahme der Academy of Medical Sciences: ,It is [...] important to clearly delineate the different ways and contexts in which this technology might be used: clearly distinguishing the use of this technology in a research context compared with its potential application in a clinical setting; as well as distinguishing the use of these technologies using somatic (non-reproductive) or germ (reproductive) cells." (The Academy of Medical Sciences 2017 [2015], S. 245).

${ }^{5}$ Das Embryonenschutzgesetz (EschG) verbietet bislang jegliche Eingriffe an Embryonen zu Forschungszwecken (EschG 1990).

${ }^{6}$ Ein solches Moratorium wird explizit wie implizit von verschiedenen (inter)nationalen Expertengremien gefordert (explizit: European Group on Ethics in Science and New Technologies (EGE) 2017 [2016], S. 242; implizit: Academy of Medical Sciences 2017 [2015], S. 245; The National Academies of Sciences 2017 [2015], S. 261).
} 
griff in die Keimbahn überhaupt in Erwägung gezogen werden kann, muss zunächst ein vertretbar niedriges Risiko dieser Intervention im Vergleich zur Erbkrankheit, die es zu vermeiden gilt, erreicht werden." (ebd., S. 8). ${ }^{7}$ In dem Risikoargument zeigt sich die Nähe zum positivistisch reduzierten Wissenschaftsideal, insofern das „Risiko“ aus einem (Noch-)Nicht-Wissen resultiert und nach mehr Forschung verlangt. Dieses utilitaristische Argument nimmt direkten Bezug auf die Art des positiven Wissens vom Gene Editing und speziell auf seine Eigenschaft, zu immer größerer Exaktheit erweitert und vertieft werden zu können.

Innerhalb des Risiko-Nutzen-Kalküls stellen die möglichen Langzeitwirkungen der therapeutischen Verfahren und der nicht absehbaren Auswirkungen des Gene Editing auf künftige Generationen ein Risiko dar. Auch für die Medizinethikerin Bettina Schöne-Seifert - Mitautorin des Diskussionspapiers der Nationalen Akademie der Wissenschaften Leopoldina - ist dies ein ernstzunehmender Einwand: „Als erstes sind da die möglichen Risiken zu nennen - in ihrer Bedeutung potenziert durch ihre Weitergabe an nachfolgende Generationen. [...] in der scientific community [steigt] offenbar das Bewusstsein für die Gefahr, dass mit der Reparatur einer Punktmutation deren mögliche, aber noch unbekannte protektive Effekte verlorengehen oder unerwünschte epigenetische Effekte einhergehen könnten. " (Schöne-Seifert 2017, S. 94) Es können zudem sogenannte Off-Target-Effekte auftreten und zu erheblichen Schäden für die (künftige) Bevölkerung führen, wenn durch das CRISPRCas9-Werkzeug an unerwarteten Stellen im Genom geschnitten wird. Ganz im Sinne des utilitaristischen Prinzips vom größtmöglichen Glück für die größtmögliche Zahl wird diesem Sicherheitsrisiko, das im Grunde die ganze Menschheit betreffen kann, der therapeutische Nutzen des Gene Editing gegenübergestellt, der vor allem in der Bekämpfung von Krankheiten besteht. Christopher Gyngell, Thomas Douglas und Julian Savulescu vertreten in Hinblick auf ein kleineres Risiko zum größeren Nutzen eine das Gene Editing befürwortende Position mit Ausblick auf ein „Ja“ zur Anwendung. Die Autoren stellen den medizinischen Nutzen des Gene Editing z. B. der riskanteren In-vitro-Fertilisation gegenüber oder berufen sich auf nützliche Aspekte für die Genforschung am Menschen (Gyngell et al. 2016, S. 2-6). ${ }^{8}$ Gemäß dem Motto „Wir wissen nur noch nicht genug!“ nimmt ihre Darstellung des Sicherheitsrisikos in Hinblick auf mögliche Keimbahnveränderungen direkten Bezug auf das positive Wissen vom Gene Editing: „In addition, there will be ways to estimate

\footnotetext{
7 Vgl. die Stellungnahme der Berlin-Brandenburgischen Akademie der Wissenschaften: „Die Zeit des Moratoriums soll genutzt werden, um experimentelle, ethische und rechtliche Fragen der Keimbahntherapie offen, transparent und kritisch zu diskutieren, um die Chancen und Risiken der Technologien für Mensch und Natur klarer zu definieren und Empfehlungen für zukünftige Regelungen zu erarbeiten." (Berlin-Brandenburgische Akademie der Wissenschaften 2017 [2015], S. 223) „Die Klärung der Frage nach den Risiken und Auswirkungen - und hier auch und vor allem die Frage nach den nicht-intendierten Auswirkungen auf das Genom - sollte Gegenstand gründlicher Erforschung von Sicherheits- und Risikoaspekten sein - ergänzend zur offenen Grundlagenforschung im Bereich Genomchirurgie, da nur so eine fachkompetente Beurteilung erfolgen kann.“ (ebd., S. 224f.).

8 Julian Savulescu argumentiert an anderer Stelle für den prospektiven, gestalterischen Gebrauch des Gene Editing, wenn er das Gene Editing durch das Prinzip „Procreative Beneficence“ rechtfertigt: „,Procreative Beneficence': couples (or single reproducers) should select the child, of the possible children they could have, who is expected to have the best life, or at least as good a life as the others, based on the relevant, available information." (Savulescu 2001, S. 413).
} 
the risk from off-target-mutations [...]. This will further reduce the risk to future generations.“" (ebd., S. 9)

Im Rahmen befürwortender Stellungnahmen zum Gene Editing finden sich mitunter auch Positionen, die sich nicht direkt am positiven Wissen vom Gene Editing orientieren. Gemeint sind Argumente, die das Gene Editing aus bestehenden anderen, nicht-wissenschaftlichen Normen rechtfertigen. So lautet etwa das liberalistische Plädoyer für die Forschungsfreiheit von Bernhard Rütsche, dass ihre Einschränkung mit geltenden, lebensweltlichen Normen konfligiert (Rütsche 2017). ${ }^{9}$ Nach Rütsche entspricht Forschung einem individuellen Bedürfnis und befördert das Gemeinwohl. „Forschungsverbote sind somit empfindliche Freiheitseingriffe. Sie sind nur dann hinnehmbar, wenn sie zum Schutz höherwertiger Güter oder Interessen unerlässlich sind. Ein hohes Gut [...] ist die Menschenwürde.“ (ebd., S. 243) Auf die Frage, ob die Menschenwürde durch die Forschung an Embryonen mittels CRISPR-Cas9-Technologie verletzt werde, schreibt er: „Während mit der Würde überzähliger Embryonen Lebewesen geschützt werden, die keinerlei Empfindungsfähigkeit oder Bewusstsein und damit keine eigenen Bedürfnisse haben, verteidigen Grundrechte von Personen wie die Forschungsfreiheit elementare Interessen, deren Verletzung als Schmerz, Leiden oder sonst wie negativ erfahren wird. Aus einem unabhängigen moralischen Standpunkt wäre es nicht zu rechtfertigen, objektive, abstrakte Werte über das subjektive Empfinden vulnerabler Menschen zu stellen. Die Würde früher Embryonen darf damit nicht absolut gesetzt und generell der Forschungsfreiheit übergeordnet werden." (ebd., S. 244) Wenn sich dieser Argumentationstypus auch nicht direkt am positiven Wissen vom Gene Editing orientiert, so ist doch festzuhalten, dass er der positivistischen Grundhaltung des Utilitarismus verpflichtet ist und somit der epistemischen Grundlage des positiven Wissens vom Gene Editing zumindest indirekt Rechnung trägt. Diese indirekte Stellungnahme wägt Risiken und Nutzen als Fakten ab. Sie orientiert sich am Nützlichkeitsprinzip, welches nicht durch abstrakte Vorstellungen oder Werte zu rechtfertigen, sondern von unserer Erfahrung bzw. in der spezifischen methodischen Einstellung auf Tatsachenwissen zu prüfen ist. „Die Frage, wie wir mit neuen Technologien umgehen sollen, muss auf der Basis von nüchternen, mit aller Sorgfalt durchgeführten Nutzen-Risiko-Analysen beantwortet werden. Nur wenn Technologien Risiken mit sich bringen, die sich nicht beherrschen lassen und die nicht durch ihren potenziellen Nutzen aufgewogen werden, sind Verbote angezeigt.“ (ebd., S. 246) ${ }^{10}$ - Sofern sich dieser letzte Argumentationstyp auf andere, nicht-wissenschaftliche Normen beruft, hat er Ähnlichkeiten zu dem

\footnotetext{
9 „Die Frage, ob es zulässig sein soll, die gezielte Veränderung des menschlichen Erbguts (,Gene Editing $\left.{ }^{*}\right)$ mittels CRISPR-Cas9-Technologie an menschlichen Embryonen zu erforschen, ist im Grunde falsch gestellt. Die Frage müsste lauten, ob für ein staatliches Verbot solcher Forschung gute Gründe vorliegen. In einem liberalen Rechtstaat sind nicht die Freiheiten der Bürgerinnen und Bürger, sondern Einschränkungen ihrer Freiheiten rechtfertigungsbedürftig. Zu diesen Freiheiten gehört die Forschungsfreiheit." (Rütsche 2017, S. 243).

${ }^{10}$ Rütsche hebt hervor, dass eine ,schleichende Ausweitung in Richtung leistungssteigernder Interventionen“ kontrolliert werden kann (ebd., S. 246). „Allerdings haben es Gesellschaft und Staat - wie bei allen potenziell gefährlichen Technologien - in der Hand, Missbrauch konsequent zu verhindern und zu sanktionieren. Wenn es für ein Verbot gentechnologischer Verbesserungen der menschlichen Natur gute Gründe gibt, da ansonsten fundamentale Werte der Gesellschaft bedroht wären, wird es auch künftig keinen Konsens für eine Lockerung dieses Verbots geben.“ (ebd.).
} 
der Gegner des Gene Editing. Der Unterschied zu den Gegnern besteht schließlich darin, dass nicht die Ausführung des Gene Editing, sondern die Einschränkung mit gegebenen Normen in Konflikt gerät.

\subsection{Ethische Einwände gegen das Gene Editing und das Paradox einer wertfreien Verbindlichkeit}

Eine argumentative Option für die Gegner des Gene Editing liegt darin, den Ansprüchen und Grundüberzeugungen der positivistischen Wissenschaftsauffassung direkt zu widersprechen, indem die mit dem Objektivitäts- und Exaktheitsideal in Aussicht gestellte hundertprozentige Sicherheit des Verfahrens sowie die Absehbarkeit und Beherrschbarkeit aller Konsequenzen angezweifelt wird. Das Wissen vom Gene Editing erscheint dann als ungewiss in Hinblick auf die Kalkulierbarkeit der Risiken, die diese Technologie in sich birgt. So wird ebenfalls betont, dass die Eingriffe in sich selbst organisierende Systeme irreversible Folgeschäden mit sich bringen könnten, deren Entwicklung nicht vollständig oder sicher kalkuliert werden kann, man denke z. B. an evolutionäre Schäden (Krohn und Krücken 1993). ${ }^{11}$ „Wir sind [...] mit Unsicherheiten unterschiedlichen Typs konfrontiert: Zum einen mit Unsicherheiten in den Aussagen über Schäden, deren Möglichkeit [...] zwar als Risiken kalkulierbar gemacht werden, in Ansehung der Einzelfälle jedoch Unsicherheitsmargen mit Blick auf die Standardabweichung zurücklassen [...]. Daneben ist die Unsicherheit über Aussagen bezüglich ihres Wahrheitsanspruches zu unterscheiden, also die Frage, ob und inwieweit die Risikokalkulation [...] überhaupt den Bezugssachverhalt erfasst [...].“ (Hubig 2015, S. 142)

Solche direkten Einwände tragen der spezifischen Art des Wissens vom Gene Editing und dessen positivistischer Grundlage Rechnung, indem sie die damit verbundenen Ansprüche und Versprechungen infrage stellen. Darüber hinaus verfügen die Gegner des Gene Editing aber vor allem über eine Reihe von Argumenten, die nicht auf die Art dieses Wissens und dessen Ansprüche reagieren, sondern bestimmte Normen in den Blick bringen, die - wie der ,Wert menschlichen Lebens“ - unabhängig von und neben den Selbstverständlichkeiten der positiven Wissenschaften bestehen und unsere Lebenswelt ebenfalls bestimmen bzw. fundamental für unser Zusammenleben und Selbstverständnis sind. Diese Normen werden nach Auffassung der Gegner durch die Forschungen zum Gene Editing und die praktische Anwendung ihrer Resultate verletzt. Die Argumente der Gegner verzeichnen aber nicht einfach nur diesen Normverstoß, sondern fokussieren die Aufweichung oder sogar den Verlust dieser Normen durch die Forschungen zum Gene Editing und die praktische Anwendung ihrer Resultate. Sie behaupten, dass diese Forschungen und Anwendungen erhebliche Auswirkungen auf die Geltung der Normen haben, die selbst

\footnotetext{
11 In einem ähnlichen Kontext betonen Roland Kipke, Martin Hähnel und Markus Rothhaar, dass die Risiken in der grundsätzlichen Lückenhaftigkeit des Wissens vom Gene Editing bestehen: „Soviel man auch [...] über die Funktionsweise einzelner Gene in der Embryonalentwicklung lernen mag - wie sich solche Veränderungen in der Keimbahn bei geborenen Menschen und deren Nachkommen auswirken, werden wir durch Studien an Embryonen niemals vollständig erfahren. Das ließe sich nur studieren, indem man die Keimbahn von Embryonen manipuliert, die implantiert, ausgetragen und geboren werden." (Kipke et al. 2017, S. 250).
} 
den Maßstab für die Beurteilung dieser Forschungen und Anwendungen abgeben. Im Folgenden werden drei Argumentationstypen gegen das Gene Editing angeführt, die unter einem bioethischen, kultur- oder sozialphilosophischen Vorzeichen auf einen solchen Abrieb und Verlust einst selbstverständlicher Normen aufmerksam machen. Jene lebensweltlichen Grundüberzeugungen stehen mit dem Wissen vom Gene Editing in radikalem Konflikt, d.h. es gelingt nicht, das positive Wissen vom Gene Editing mit diesen normativen Grundsätzen und Selbstverständlichkeiten des alltäglichen Lebens in Einklang zu bringen.

Das sogenannte Dammbruchargument, das ebenso unter dem Namen „Argument der schiefen Ebene“ oder ,slippery-slope-argument“ bekannt ist, besagt, dass eine bestimmte Handlung, die für sich unproblematisch sein mag, weitere Handlungen nahelegen könnte, die entweder selbst oder hinsichtlich ihrer Folgen als ethisch bedenklich erachtet werden müssen. Die Befürchtung ist, dass kein strenges Kriterium mehr ausfindig gemacht werden kann, durch das die eine Handlung als vertretbar und die andere als unannehmbar begründet werden kann (Düber und Rojek 2015, S. 9). Verweise auf die wunscherfüllende Medizin oder das Designerbaby sind typisch für ein Dammbruch-Szenario. So besteht das Problematische am Gene Editing ,in dem, was damit möglich und wahrscheinlich wird: beliebige Eingriffe in die menschliche Keimbahn, die irreversible Weitergabe der Änderungen an zukünftige Generationen, gravierende Verschiebungen im Verhältnis der Generationen untereinander, ethisch fragwürdige klinische Tests und die Verwischung der Speziesgrenzen." (Kipke et al. 2017, S. 250) $)^{12}$

Das Wissen vom Gene Editing wird häufig als normabweichend charakterisiert, sofern seine Anwendungsvisionen mit Konzeptionen der Menschenwürde in Konflikt stehen, (künftige) Menschen in diesen Anwendungsvisionen also instrumentalisiert werden. „So wird die Meinung vertreten, dass etwa das Austragen von Kindern für andere Person [sic], die sog. Leihmutterschaft sowie die Forschung an Kindern und Embryonen, die Eizellspende von Frauen im Kontext künstlicher Befruchtung (IVF), oder auch der Verkauf von Organen eine solche Instrumentalisierung darstelle. All diese Praktiken, so wird argumentiert, sind als unzulässig anzusehen, weil mit ihnen Menschen bloß als Mittel behandelt werden. [...] Bloß als Mittel wird man nach Kant folglich behandelt, wenn man von anderen in einer Weise behandelt wird, in die man nicht einwilligen kann; gleichzeitig wird man dann auch nicht als Zweck an sich selbst behandelt.“ (Schaber 2015, S. 102) - Die Gefahr besteht also darin, dass das durch die Forschungen auf dem Feld des Gene Editing bereitgestellte und vermeintlich wertfreie, technische Wissen selbst wiederum auf eine Weise verwendet wird, durch die einzelne Menschen für fragliche Zwecke verfügbar werden. Es wäre z. B. das Stigma denkbar, dass künftig die Nichtbeachtung der genetischen Beschaffenheit eines Embryos und die versäumte genetische Korrektur mit fehlendem Risikobewusstsein gleichgesetzt werden. Vor der Gefahr der Instrumentalisierung warnt auch Jürgen Habermas. Er hebt hervor, dass durch das Gene Editing bei ei-

\footnotetext{
12 Was die Wahrscheinlichkeit des Eintretens jener Dammbrüche betrifft, so betonen die Autoren, dass „,die befürchtete weitergehende Anwendung des Gene Editing [...] in der Logik dieser Forschung bereits angelegt [ist]. Es ergibt kaum einen Sinn, Gene Editing an Embryonen zu erforschen, ohne das später auch praktizieren zu wollen. Die Annahme, es bleibe langfristig bei dieser Forschung, ist naiv.“ (ebd., S. 251 f.).
} 
nem Embryo ein völlig neues Verhältnis zum eigenen Leib für die sich aus diesem Embryo entwickelnde Person gestiftet wird. Die beliebige Verteilung der Talente und Eigenschaften durch die unvorhersehbare Natur wird ausgeschaltet zugunsten eines instrumentellen Handelns von Menschen an Menschen. Anders als z. B. pädagogische Maßnahmen sind die Eingriffe durch das Gene Editing endgültig und können kommunikativ nicht mehr eingeholt werden. ,[M]it der Entscheidung über sein genetisches Programm haben die Eltern Absichten verbunden, die sich später in Erwartungen an das Kind verwandeln, ohne jedoch dem Adressaten die Möglichkeit zu einer revidierenden Stellungnahme einzuräumen. [...] Die Eltern haben ohne Konsensunterstellung allein nach eigenen Präferenzen so entschieden, als verfügten sie über eine Sache.“ (Habermas 2001, S. 90)

Ein weiterer Argumentationstyp, der das Wissen vom Gene Editing als normübergreifend thematisiert, hebt den Wert der menschlichen Natur hervor. ${ }^{13}$ Damit gehen nicht selten Überlegungen über die Verwischung unserer Speziesgrenzen einher. So schreibt der Politikwissenschaftler Francis Fukuyama in seinem wissenschaftskritischen Buch „Vom Ende des Menschen“: „Wir sind möglicherweise dabei, in eine nachmenschliche Zukunft einzutreten, in der die Technologie uns die Fähigkeit verleiht, diesen Wesenskern im Laufe der Zeit langsam aber sicher zu verändern. Viele Menschen greifen unter der Fahne der menschlichen Freiheit nach dieser Macht.“ (Fukujama 2002, S. 300) Mögliche Folgen der Verwischung unserer Speziesgrenzen können z. B. unsere Vorstellung von natürlicher Gleichheit betreffen, welche die Grundlage vieler unserer Rechtskodizes darstellt. Wie steht es dann etwa noch um die gerechte Verteilung von Gütern? In diesem Kontext warnt Michael J. Sandel davor, die ,Wertschätzung des Charakters menschlicher Fähigkeiten und Erfolge als Gabe aus[zu]höhl[en]“ (Sandel 2008, S. 26), denn ,,[d]ie natürlichen Talente, die es den Erfolgreichen gestatten zu florieren, sind nicht ihr eigenes Werk, sondern vielmehr Glück - ein Ergebnis der genetischen Lotterie. Wenn unsere genetische Ausstattung eine Gabe ist [...], ist es ein Fehler zu glauben, wir hätten ein Anrecht auf das volle Maß des Gewinns, den sie in einer Marktwirtschaft erzielt. Wir haben daher eine Verpflichtung, diesen Gewinn mit denen zu teilen, denen ohne eigenes Verschulden vergleichbare Begabungen fehlen.“ (ebd., S. 29)

Solche normverändernden Wirkungen des wissenschaftlichen Forschens sind von einer positivistisch orientierten Auffassung dieses Wissens, wie sie oben den Befürwortern des Gene Editing unterstellt wurde, nicht nachzuvollziehen. Die dort aufgeführten Positionen halten allesamt das nach wissenschaftlichen Kriterien gewonnene objektive Wissen vom Gene Editing für unabhängig von seiner praktischen Anwendung. Dieses Wissen steht für den praktischen Gebrauch zur Verfügung und kann zum guten Nutzen der Menschen eingesetzt werden. Es kann aber genauso gut zurückgehalten werden, wenn es dafür entsprechende Gründe gibt. Von dieser Auffassung unbemerkt bleibt, dass die vermeintlich objektive Realität auf einer be-

\footnotetext{
13 ,Natürlichkeit scheint neben dem Kriterium der Gesundheitsorientierung das wichtigste implizite Kriterium, nach dem die Akzeptabilität biomedizinischer Verfahren beurteilt wird. Das heißt, soweit gentechnische und reproduktionsmedizinische Verfahren der Therapie und Präventionen von Krankheiten dienen oder Defizite (wie ungewollte Kinderlosigkeit) kompensieren, gelten sie weitestgehend als akzeptabel, weniger jedoch, wenn sie zu anderen Zwecken genutzt werden, etwa zur Steigerung von Fähigkeiten oder zur qualitativen Auswahl der Nachkommen." (Birnbacher 2011, S. 150).
} 
stimmten methodischen Einstellung beruht, die ihrerseits durch das positivistische Weltbild als verbindlich eingefordert wird. Das objektive neutrale Wissen beruht somit selbst auf bestimmten Normen und Werten, die im Rahmen dieser eben neutralen Wissensordnung grundsätzlich nicht reflektiert werden können. Das ist das Paradox einer verbindlichen Wertfreiheit.

Aus demselben Grund, aus dem die eigenen normativen Grundlagen nicht nachvollzogen werden können, kann auch auf die nicht-wissenschaftlichen Normen, auf welche die Gegner des Gene Editing rekurrieren, nicht eingegangen werden, die häufig als abstrakt oder kulturrelativ abgetan werden (The Hinxton Group 2017 [2015], S. 251). Auf der Grundlage des positivistischen Weltbildes ist es insgesamt nicht möglich, den angedeuteten Prozess einer Normveränderung zu berücksichtigen weder in Hinblick auf den befürchteten Verlust bisheriger ethischer Selbstverständlichkeiten noch in Hinblick auf die weitere Verfestigung des positivistischen Weltbildes bzw. die Beurteilung des Gene Editing auf dieser Grundlage. Symptomatisch dafür ist etwa die Forderung nach einer Revision der Gesetzeslage, die der aktuellen ethischen Beurteilung längst nicht mehr angemessen sei. ${ }^{14}$

\section{Das Wissen vom Gene Editing als prekäres Wissen}

Um die vorangegangenen Überlegungen zu dem besonderen epistemischen Status des Wissens vom Gene Editing in einem Begriff zu bündeln, bietet es sich an dieses als ,prekäres Wissen“ zu kennzeichnen. Einerseits erfüllt es die Kriterien, die Wittgenstein an den Begriff des positiven Wissens stellt, denn es stehen allgemein anerkannte Verfahren zur Verfügung, nach denen sich dieses Wissen begründen lässt, nämlich die Verfahren der positiven Wissenschaften. Sie sind in einem positivistisch orientieren Weltbild verankert, das, wie mit Husserl deutlich geworden ist, selbst im außerwissenschaftlichen, lebensweltlichen Kontext selbstverständlich gilt. In dieser Hinsicht entspricht das Wissen vom Gene Editing geltenden Normen. Andererseits widerstreitet dieses Wissen anderen, nicht-wissenschaftlichen Normen, die ebenso

\footnotetext{
14 Schöne-Seifert spricht sich z.B. dafür aus, eine offene und informierte Diskussion zu führen, denn es stünde ,viel auf dem Spiel: zu viel, um an eingefahrenen Positionen festzuhalten.“ (Schöne-Seifert 2017, S. 96) Sie betont, dass die ethischen Argumente innerhalb der aktuellen Debatte um therapeutische Keimbahneingriffe bereits seit den 1980er Jahren im Umlauf sind und es sich sicher lohne, sie wieder hervorzuholen, aber es „scheinen auch neue Blicke auf die alten Argumente angebracht.“ (ebd., S. 94) Vor allem die restriktive Regelung des Embryonenschutzgesetzes in Deutschland sei zu überdenken, weil sie die Forschung an menschlichen Keimzellen erheblich einschränke: „Hochrangige Erkenntnisgewinne über die menschliche Embryonalentwicklung, über gesunde und gestörte Genfunktionen sowie über deren medizinische Beeinflussungsmöglichkeit zu erzielen, bleibt damit ausländischen Forschern vorbehalten, mit denen deutsche Wissenschaftler auch nicht kooperieren dürfen. Das alles wäre offenkundig ein unbedingt zu zahlender ,Preis“, wenn die hinter dem Embryonenschutzgesetz stehende Position des vollen Würdeund Lebensschutzes bereits für befruchtete menschliche Eizellen ihrerseits einleuchtend begründbar wäre. Doch wird sie in einer säkularisierten und biologisch informierten Gesellschaft wohl eher zunehmend strittig und ist nach Ansicht mancher daher politisch erneut zur Diskussion zu stellen. Die u. U. provozierende Diagnose, das Embryonenschutzgesetz sei nicht mehr ,zeitgemäß‘, muss also keineswegs ein ,Einknicken“ der Ethik vor den Interessen von Forschern oder künftigen Patienten zum Ausdruck bringen, sondern vielmehr die fragliche Überzeugungskraft einer ethischen Prämisse, die in neuem Licht zu diskutieren wäre." (ebd., S. 95).
} 
in unserer Lebenswelt verankert und für unser Selbstverständnis und Zusammenleben fundamental sind. Dieser Konflikt besteht seit mehr als dreißig Jahren und auch die meisten Argumente für und gegen das Gene Editing sind ebenso lange bekannt. Trotz dieses Patts auf der argumentativen Ebene ist der Konflikt durch eine innere Dynamik bestimmt, insofern der wissenschaftliche Fortschritt einerseits neue Fakten schafft, ${ }^{15}$ während andererseits die diesen Fakten widersprechenden, aber etablierten Selbstverständlichkeiten zunehmend zerrieben $\mathrm{zu}$ werden scheinen. Zumindest teilen diese Sorge vor einem Verlust bisher selbstverständlicher Normen die meisten Gegner des Gene Editing.

In diesem Kontext wurde auf einen aktuellen Normalisierungs- und Normierungsprozess aufmerksam gemacht, der bisher kaum erforscht und auch in seiner ethischen Bedeutung nicht hinreichend reflektiert worden ist. Die vorliegende Betrachtung der epistemischen Grundlagen konnte allenfalls einige Hinweise dafür liefern, denen in weiteren Forschungen nachgegangen werden müsste. Daraus lassen sich ebenfalls Impulse für die ins Stocken geratene Debatte zum Gene Editing erwarten.

Open Access Dieser Artikel wird unter der Creative Commons Namensnennung 4.0 International Lizenz (http://creativecommons.org/licenses/by/4.0/deed.de) veröffentlicht, welche die Nutzung, Vervielfältigung, Bearbeitung, Verbreitung und Wiedergabe in jeglichem Medium und Format erlaubt, sofern Sie den/die ursprünglichen Autor(en) und die Quelle ordnungsgemäß nennen, einen Link zur Creative Commons Lizenz beifügen und angeben, ob Änderungen vorgenommen wurden.

\section{Literatur}

\section{Zeitschriftenartikel}

Berlin-Brandenburgische Akademie der Wissenschaften. 2017 [2015]: Genomchirurgie beim Menschen Zur verantwortlichen Bewertung einer neuen Technologie. Eine Analyse der Interdisziplinären Arbeitsgruppe Gentechnologiebericht. In: Jahrbuch für Wissenschaft und Ethik 21: 223-239.

European Group on Ethics in Science and New Technologies (EGE). 2017 [2016]: Statement on Gene Editing. In: Jahrbuch für Wissenschaft und Ethik 21: 241-243.

Kipke, Roland; Hähnel, Martin; Rothhaar, Markus. 2017. Contra: Soll das sogenannte „Gene Editing“ mittels CRISPR/Cas9-Technologie an menschlichen Embryonen erforscht werden? In: Ethik in der Medizin 29: 249-252.

Rütsche, Bernhard. 2017. Pro: Soll das sogenannte „Gene Editing“ mittels CRISPR-Cas9-Technologie an menschlichen Embryonen erforscht werden? In: Ethik in der Medizin 29: 243-247.

Sandel, Michael J. 2008. Beherrschung und Gabe, Übers. Rudolf Teuwsen. In: Die politische Meinung 467: 26-32.

Savulescu, Julian. 2001. Procreative Beneficence: Why we Should Select the Best Children. In: Bioethics 15 5/6: 413-426.

Schöne-Seifert, Bettina. 2017: Genscheren-Forschung an der menschlichen Keimbahn: Plädoyer für eine neue Debatte auch in Deutschland. In: Ethik in der Medizin 29: 93-96.

The Hinxton Group. 2017 [2015]. Statement on Genome Editing Technologies and Human Germline Genetic Modification. In: Jahrbuch für Wissenschaft und Ethik 21: 249-257.

The National Academies of Sciences, Engineering and Medicine (USA). 2017 [2015]. On Human Gene Editing: International Summit Statement. In: Jahrbuch für Wissenschaft und Ethik 21: 259-261.

15 Am 26.11.2018 verkündet die Tagespresse die Meldung, dass der chinesische Forscher He Jiankui das Genom zweier noch ungeborener Kinder mithilfe von CRISPR-Cas9 manipuliert habe, um sie gegen das HI-Virus resistent zu machen. Die Kinder kamen zur Welt. Damit hat er die erste Geburt genmanipulierter Babys veranlasst - ohne Rücksicht auf ethische Stellungnahmen oder Warnungen (deutschlandfunk.de 2018). 
The Academy of Medical Sciences, Association of Medical Research Charities, Cancer Research UK, Biotechnology and Biological Sciences Research Council, Medical Research Council, Progress Educational Trust, Wellcome Trust, Wellcome Trust Sanger Institute (UK). 2017 [2015]. Genome Editing in Human Cells - Initial Joint Statement. In: Jahrbuch für Wissenschaft und Ethik 21: 245-247.

\section{Online-first-Artikel}

Gyngell, Christopher; Douglas, Thomas; Savulescu, Julian. 2016. The Ethics of Germline Gene Edition. Journal of Applied Philosophy, https://doi.org/10.1111/japp.12249.

Ma, Hong u. a. 2017. Correction of a pathogenic gene mutation in human embryo. Nature, https://doi.org/ 10.1038 /nature23305.

\section{Buch}

Fukujama, Francis. 2002. Das Ende des Menschen, Übers. Klaus Kochmann. Stuttgart, München/Deutsche Verlagsanstalt.

Habermas, Jürgen. 2001. Die Zukunft der menschlichen Natur. Frankfurt a.M./Suhrkamp.

Husserl, Edmund. ${ }^{2} 1962$ [1954]. Die Krisis der europäischen Wissenschaften und die transzendentale Phänomenologie. Eine Einleitung in die phänomenologische Philosophie. Husserliana. Gesammelte Werke. Bd. 6, Hrsg. Walter Biemel. Den Haag, Martinus Nijhoff.

Kober, Michael. 1993. Gewißheit als Norm. Wittgensteins erkenntnistheoretische Untersuchungen in „Über Gewißheit“. Berlin, New York/Walter de Gruyter.

Krebs, Andreas. 2007. Worauf man sich verlässt. Sprach- und Erkenntnisphilosophie in Ludwig Wittgensteins „Über Gewißheit“. Würzburg/Könighausen und Neumann.

Wittgenstein, Ludwig. 1984 [1970]. Über Gewißheit. Werkausgabe 8. Frankfurt a.M./Suhrkamp.

\section{Buchkapitel}

Bermes, Christian, 2017. Die Krisis der europäischen Wissenschaften und die transzendentale Phänomenologie. In Husserl-Handbuch, Hrsg. Sebastian Luft, Maren Wehrle, 97-104. Stuttgart/J. B. Metzler.

Birnbacher, Dieter. 2011. Natürlich/künstlich. In Handbuch Angewandte Ethik, Hrsg. Ralf Stoecker, Christian Neuhäuser, Marie-Luise Raters, 148-152. Stuttgart, Weimar/J. B. Metzler.

Düber, Dominik; Rojek, Tim. 2015. Argument der schiefen Ebene. In Handbuch Bioethik, Hrsg. Dieter Sturma, Bert Heinrichs, 9-13. Stuttgart, Weimar/J. B. Metzler.

Hubig, Christoph. 2015. Risiko. In Handbuch Bioethik, Hrsg. Dieter Sturma, Bert Heinrichs, 140-147. Stuttgart, Weimar/J. B. Metzler.

Knabenschuh de Porta, Sabine. 2013: Phänomenologie versus phänomenologische Probleme. Die parallaktische Lösung Wittgensteins. In Phenomenology as Grammar, Hrsg. Jesús Padilla Gálvez, 169183. Frankfurt a.M./Ontos.

Krohn, Wolfgang; Krücken, Georg. 1993: Risiko als Konstruktion und Wirklichkeit. In Riskante Technologien: Reflexion und Regulation, Hrsg. Dies., 9-44. Frankfurt a.M./Suhrkamp.

Peursen, Cornelius Anthonie van. 1965: Übereinkunft und Gegensatz im heutigen europäischen Denken. Edmund Husserl und Ludwig Wittgenstein (Rektoratsrede, gehalten am 18.7.1964). In Ludwigs-Maximilians-Universität. Niederländische Gelehrtenwoche, 121-132. München.

Schaber, Peter. 2015. Menschenwürde und Instrumentalisierung. In Handbuch Bioethik, Hrsg. Dieter Sturma, Bert Heinrichs, 102-108. Stuttgart, Weimar/J. B. Metzler.

Wiesemann, Claudia. 2011. Eltern und Kinder. In Handbuch Angewandte Ethik, Hrsg. Ralf Stoecker, Christian Neuhäuser, Marie-Luise Raters, 242-248. Stuttgart, Weimar/J. B. Metzler. 


\section{Online Dokument}

deutschlandfunk.de. 02. Dezember 2018 Der Geist ist aus der Flasche. https://www.deutschlandfunk.de/ genmanipulation-an-embryonen-der-geist-ist-aus-der-flasche.720.de.html?dram:article_id=434780. Zugegriffen: 03.12.2018.

EschG. 13. Dezember 1990. https:/www.gesetze-im-internet.de/eschg/BJNR027460990.html. Zugegriffen: 17.07.2019.

Nationale Akademie der Wissenschaften Leopoldina. 2017. Ethische und rechtliche Beurteilung des genome editing in der Forschung an humanen Zellen. https://www.leopoldina.org/uploads/tx_ leopublication/2017_Diskussionspapier_GenomeEditing.pdf. Zugegriffen: 17.07.2019.

Orth, Ernst Wolfgang. 2000. ,Lebenswelt“ als eine unendliche Illusion? Der Lebensweltbegriff und seine unendlichen Weiterungen. In: Protosoziologie im Kontext. „Lebenswelt“ und „System“ in Philosophie und Soziologie, hg. Von Gerhard Preyer, Georg Peter, Alexander Ulfig (Frankfurt a. Main) 28-40. https://www.google.com/url?sa=t\&rct=j\&q=\&esrc=s\&source=web\&cd=1\& ved=2ahUKEwi4tY2Y6trjAhVlqnEKHa5TANkQFjAAegQIARAC\&url=https\%3A\%2F\%2F humanities.verlags-shop.de\%2Findex.php\%3Fcontroller\%3Dattachment\%26id_attachment\%3D8\& usg=AOvVaw2HDdSyVQWWdLLQsuOuckvn. Zugegriffen: 17.07.2019.

Spiegel Online. 12. April 2018: Baby vier Jahre nach Tod seiner Eltern geboren. http://www.spiegel. de/gesundheit/schwangerschaft/china-baby-vier-jahre-nach-tod-seiner-eltern-geboren-a-1202643. html. Zugegriffen: 17.07.2019. 